01

\title{
Радиационные константы в спектре иона W VII
}

\author{
() А.В. Логинов, В.И. Никитченко \\ Петербургский государственный университет путей сообщения, \\ 190031 Санкт-Петербург, Россия \\ e-mail: andrlgnv@yandex.ru
}

Поступила в редакцию 23.03.2020 г.

В окончательной редакции 23.03.2020 г.

Принята к публикации 15.04.2020 г.

Полуэмпирическим методом промежуточной связи с использованием экспериментальных уровней энергии, известных из литературы, в электродипольном приближении рассчитаны радиационные вероятности переходов $4 f^{13} 5 p^{6} 6 p+4 f^{14} 5 p^{5} 6 p-4 f^{13} 6 s, 4 f^{13} 7 s$ и времена жизни уровней $4 f^{13} 7 s$ в спектре эрбийподобного иона W VII. Радиальные интегралы переходов, необходимые для вычисления абсолютных значений вероятностей переходов, получены в форме длины с функциями Хартри-Фока.

Ключевые слова: вероятности радиационных переходов, времена жизни уровней, полуэмпирический метод, изоэлектронный ряд эрбия.

DOI: $10.21883 /$ OS.2020.08.49700.113-20

\section{Введение}

Мотивом для выполнения настоящего расчета послужили два фактора. Во-первых, согласно базе данных [1], какие-либо опубликованные данные по радиационным константам в спектре иона W VII отсутствуют. Во-вторых, ранее [2,3] нами были вычислены вероятности переходов и времена жизни уровней в спектрах ионов Yb III-Ta VI изоэлектронного ряда эрбия, к которому принадлежит ион W VII. Таким образом, настоящий расчет можно рассматривать как продолжение работ [2,3]. Рассмотрены электродипольные переходы между четными уровнями $4 f^{13} 5 p^{6} 6 p+4 f^{14} 5 p^{5} 6 p$ и нечетными $4 f^{13} 6 s$, $4 f^{13} 7 s$.

\section{Метод расчета}

Волновые функции промежуточной связи нечетных уровней $4 f^{13} 6 s, 4 f^{13} 7 s$, необходимые для вычисления вероятностей переходов, найдены в одноконфигурационном приближении. При вычислении волновых функций промежуточной связи четных уровней принято во внимание наложение конфигураций $4 f^{13} 5 p^{6} 6 p+4 f^{14} 5 p^{5} 6 p$. Радиальные интегралы, входящие в выражения для матричных элементов оператора энергии, получены методом наименьших квадратов (МНК) по известным экспериментальным значениям [4] уровней энергии. Отметим, что эти значения фигурируют как рекомендованные в базе данных [1]. Приняты во внимание электростатическое, спин-орбитальное и так называемое эффективное взаимодействия. Соответствующие величины обозначены в табл. 1, 2 как $F_{f l}^{k}, G_{f l}^{k}$ (электростатические интегралы Слэтера прямого и обменного взаимодействий), $\xi_{4 f}, \xi_{6 p}$ (спин-орбитальные константы), $F_{1}$ (интеграл
Слэтера прямого взаимодействия с запрещенным рангом, „эффективно“ учитывающий вклад двухчастичных взаимодействий, операторы которых действуют только на пространственные координаты). Правила вычисления угловых коэффициентов перед параметрами $F_{f l}^{k}, G_{f l}^{k}$, $\xi_{4 f}, \quad \xi_{6 p}$ общеизвестны (например, [5]), правила вычисления угловых коэффициентов перед эффективным параметром $F_{1}$ можно найти в [3]. Здесь только напомним, что для описания межэлектронных взаимодействий в конфигурациях типа $f^{13} l, p^{5} p$ (дырка-электрон) достаточно привлечь одно- и двухчастичные операторы.

Качество реализации предписания наименьших квадратов определяется дисперсиями параметров, а также стандартными $(\sigma)$ и среднеквадратичными $(\Delta)$ отклонениями по энергии:

$$
\begin{gathered}
\sigma=\sqrt{\left.\sum_{i=1}^{n}\left(E_{\mathrm{calc}}^{i}-E_{\mathrm{exp}}^{i}\right)^{2} /(n-m)\right),} \\
\Delta=\sqrt{\left.\sum_{i=1}^{n}\left(E_{\mathrm{calc}}^{i}-E_{\mathrm{exp}}^{i}\right)^{2} / n\right)},
\end{gathered}
$$

где $n$ - число экспериментальных уровней, включенных в процедуру МНК, $m$ - число свободно варьируемых параметров, $E_{\text {calc }}^{i}, E_{\exp }^{i}-$ соответственно вычисленное и экспериментальное значения энергии $i$-го уровня.

Из табл. 1, 2 видно, что все параметры хорошо определены. Сопоставление с соответствующими величинами из $[2,3]$ для $\mathrm{Yb}$ III-Ta VI показывает также, что эти параметры (исключая $F_{1}$ ) монотонно меняются с изменением заряда ядра. Отметим, что учет наложения конфигураций $4 f^{13} 5 p^{6} 6 p+4 f^{14} 5 p^{5} 6 p$ 
Таблица 1. Параметры (в $\left.\mathrm{cm}^{-1}\right)$ матрицы энергии конфигураций $4 f^{13} 6 s, 4 f^{13} 7 s$

\begin{tabular}{c|c|c}
\hline Параметр & $4 f^{13} 6 s$ & $4 f^{13} 7 s$ \\
\hline$F_{f s}^{0}$ & $34605 \pm 8$ & $52702 \pm 3$ \\
\hline$G_{f s}^{3}$ & $5229 \pm 118$ & $1800 \pm 40$ \\
\hline$\xi_{4 f}$ & $4960 \pm 5$ & $4966 \pm 2$ \\
\hline$\sigma$ & 17 & 6 \\
\hline$\Delta$ & 8 & 3
\end{tabular}

заметно уменьшил стандартное отклонение по энергии - от $197 \mathrm{~cm}^{-1}$ для $4 f^{13} 5 p^{6} 6 p$ и $210 \mathrm{~cm}^{-1}$ для $4 f^{14} 5 p^{5} 6 p$ (в одноконфигурационном приближении) до $40 \mathrm{~cm}^{-1}$. Однако на вероятностях переходов указанное наложение конфигураций сказалось не очень заметно, а значения времен жизни уровней $4 f^{13} 7 s$, определяемые наиболее интенсивными переходами, совпадают при расчете в одно- и многоконфигурационном приближениях с точностью до четырех значащих цифр.

\section{Результаты и обсуждение}

Полученные функции промежуточной связи использованы далее для расчета вероятностей электродипольных переходов, обозначенных в табл. 3, 4 как $A$, и сил осцилляторов $g f$. При этом радиальные интегралы переходов найдены в форме длины с радиальными функциями, рассчитанными методом Хартри-Фока по программе [6]. Суммированием вероятностей переходов найдены времена жизни уровней $4 f^{13} 7 s$. Для идентификации уровней в табл. 3, 4 принята система обозначений $J_{1} j$-связи: уровни обозначаются тремя числами $\left(J_{1} j\right) J$, где $J_{1}$ - полный угловой момент электронной оболочки $f^{13}, j-$ полный угловой момент $s$ - или $p$ электрона, $J-$ полный угловой момент конфигурации $f^{13} l(l=s, p)$. Эта схема связи хорошо выполняется [4] для рассмотренных уровней, поэтому именно она использована в [4] для их идентификации. В табл. 3, 4 для удобства пользования приведены также длины волн переходов - экспериментальные из [4] и вычисленные (заключены в квадратные скобки) в настоящей работе для тех переходов, для которых экспериментальные значения отсутствуют.

В табл. 3, 4 отсутствуют переходы с участием четных уровней $4 f^{14} 5 p^{5} 6 p$. Заметим, что в рассмотренном приближении эти переходы возможны только в результате перемешивания $4 f^{13} 5 p^{6} 6 p+4 f^{14} 5 p^{5} 6 p$. Поскольку это перемешивание не очень значительно, вероятности переходов $4 f^{14} 5 p^{5} 6 p-4 f^{13} 6 s, 4 f^{13} 7 s$ невелики в сравнении с приведенными в табл. 3, 4 вероятностями переходов $4 f^{13} 5 p^{6} 6 p-4 f^{13} 6 s, 4 f^{13} 7 s$. По этой причине (дабы не перегружать таблицы) вероятности переходов $4 f^{14} 5 p^{5} 6 p-4 f^{13} 6 s, 4 f^{13} 7 s$ не приведены.

В заключение приведем значения времен жизни $\tau$ (в ns) уровней $4 f^{13} 7 s$, полученные суммированием вероятностей переходов $4 f^{13} 7 s \rightarrow 4 f^{13} 5 p^{6} 6 p+$ $+4 f^{14} 5 p^{5} 6 p: \quad \tau[(5 / 2,1 / 2) 2]=1.191, \quad \tau[(7 / 2,1 / 2) 3]=$ $=1.199, \tau[(5 / 2,1 / 2) 3]=1.198, \tau[(7 / 2,1 / 2) 4]=1.191$, и отметим (ссылаясь на [1]), что сравнивать представленные результаты пока не с чем.

\section{Благодарности}

Авторы благодарят А.Н. Рябцева (Институт спектроскопии РАН) за полезные консультации.

Таблица 2. Параметры (в $\left.\mathrm{cm}^{-1}\right)$ матрицы энергии конфигураций $4 f^{13} 5 p^{6} 6 p+4 f^{14} 5 p^{5} 6 p$

\begin{tabular}{|c|c|c|c|c|c|}
\hline Параметр & $4 f^{13} 5 p^{6} 6 p$ & Параметр & $4 f^{14} 5 p^{5} 6 p$ & Параметр & $4 f^{13} 5 p^{6} 6 p+4 f^{14} 5 p^{5} 6 p$ \\
\hline$F_{f p}^{0}$ & $41455 \pm 11$ & $F_{p p}^{0}$ & $113796 \pm 18$ & $R^{2}(5 p, 6 p ; 4 f, 6 p)$ & $-9265 \pm 304$ \\
\hline$F_{f p}^{2}$ & $12676 \pm 143$ & $F_{p p}^{2}$ & $21974 \pm 370$ & $R^{2}(5 p, 6 p ; 6 p, 4 f)$ & $-2937 \pm 970$ \\
\hline$G_{f p}^{2}$ & $3783 \pm 105$ & $G_{p p}^{0}$ & $3855 \pm 17$ & $\sigma$ & 40 \\
\hline$G_{f p}^{4}$ & $3926 \pm 280$ & $G_{p p}^{2}$ & $10233 \pm 298$ & \multirow[t]{4}{*}{$\Delta$} & \multirow[t]{4}{*}{23} \\
\hline$\xi_{4 f}$ & $4967 \pm 8$ & $\xi_{5 p}$ & $57597 \pm 20$ & & \\
\hline \multirow[t]{2}{*}{$\xi_{6 p}$} & \multirow[t]{2}{*}{$14130 \pm 19$} & $\xi_{6 p}$ & $14224 \pm 21$ & & \\
\hline & & $F_{1}$ & $-40 \pm 17$ & & \\
\hline
\end{tabular}


Таблица 3. Длины волн $(\lambda, \mathrm{nm})$, вероятности $\left(A, \mathrm{~s}^{-1}\right)$ и силы осцилляторов $(g f)$ переходов $4 f^{13} 6 p \rightarrow 4 f^{13} 6 s$ в спектре W VII

\begin{tabular}{|c|c|c|c|c|}
\hline $4 f^{13} 6 p$ & $4 f^{13} 6 s$ & $\lambda_{\exp }[4], \lambda_{\text {calc }}^{*}, \mathrm{~nm}$ & $A, \mathrm{~s}^{-1}$ & $g f$ \\
\hline$(5 / 2,3 / 2) 1$ & $(5 / 2,1 / 2) 2$ & 104.9333 & $3.39+9^{* *}$ & 1.679 \\
\hline$(7 / 2,3 / 2) 2$ & $\begin{array}{l}(5 / 2,1 / 2) 2 \\
(7 / 2,1 / 2) 3 \\
(5 / 2,1 / 2) 3\end{array}$ & $\begin{array}{l}125.2168 \\
103.7327 \\
126.2511\end{array}$ & $\begin{array}{l}3.37+7 \\
3.32+9 \\
6.33+7\end{array}$ & $\begin{array}{l}0.040 \\
2.680 \\
0.076\end{array}$ \\
\hline$(5 / 2,1 / 2) 2$ & $\begin{array}{l}(5 / 2,1 / 2) 2 \\
(7 / 2,1 / 2) 3 \\
(5 / 2,1 / 2) 3\end{array}$ & $\begin{array}{l}130.0940 \\
107.0580 \\
131.2109\end{array}$ & $\begin{array}{l}2.66+8 \\
1.42+8 \\
1.40+9\end{array}$ & $\begin{array}{l}0.337 \\
0.122 \\
1.809\end{array}$ \\
\hline$(5 / 2,3 / 2) 2$ & $\begin{array}{l}(5 / 2,1 / 2) 2 \\
(7 / 2,1 / 2) 3 \\
(5 / 2,1 / 2) 3\end{array}$ & $\begin{array}{c}101.9322 \\
{[87.210]} \\
102.6169\end{array}$ & $\begin{array}{l}3.12+9 \\
1.60+7 \\
5.94+8\end{array}$ & $\begin{array}{l}2.427 \\
0.009 \\
0.469 \\
\end{array}$ \\
\hline$(7 / 2,3 / 2) 3$ & $\begin{array}{l}(5 / 2,1 / 2) 2 \\
(7 / 2,1 / 2) 3 \\
(5 / 2,1 / 2) 3 \\
(7 / 2,1 / 2) 4\end{array}$ & $\begin{array}{l}124.5772 \\
103.2935 \\
125.6008 \\
102.4069\end{array}$ & $\begin{array}{l}4.75+7 \\
2.53+9 \\
3.31+7 \\
4.37+8 \\
\end{array}$ & $\begin{array}{l}0.077 \\
2.836 \\
0.055 \\
0.481\end{array}$ \\
\hline$(7 / 2,1 / 2) 3$ & $\begin{array}{l}(5 / 2,1 / 2) 2 \\
(7 / 2,1 / 2) 3 \\
(5 / 2,1 / 2) 3 \\
(7 / 2,1 / 2) 4\end{array}$ & $\begin{array}{l}{[171.358]} \\
133.5899 \\
{[173.355]} \\
132.1113\end{array}$ & $\begin{array}{l}1.13+5 \\
2.76+8 \\
1.03+6 \\
1.42+9 \\
\end{array}$ & $\begin{array}{c}3 \cdot 10^{-4} \\
0.515 \\
0.003 \\
2.596 \\
\end{array}$ \\
\hline$(5 / 2,3 / 2) 3$ & $\begin{array}{l}(5 / 2,1 / 2) 2 \\
(7 / 2,1 / 2) 3 \\
(5 / 2,1 / 2) 3 \\
(7 / 2,1 / 2) 4 \\
\end{array}$ & $\begin{array}{c}100.9883 \\
{[86.514]} \\
101.6603 \\
{[85.903]} \\
\end{array}$ & $\begin{array}{l}1.49+9 \\
5.56+6 \\
2.30+9 \\
3.42+6 \\
\end{array}$ & $\begin{array}{l}1.593 \\
0.004 \\
2.494 \\
0.003 \\
\end{array}$ \\
\hline$(5 / 2$ & $\begin{array}{l}(5 / 2,1 / 2) 2 \\
(7 / 2,1 / 2) 3 \\
(5 / 2,1 / 2) 3 \\
(7 / 2,1 / 2) 4 \\
\end{array}$ & $\begin{array}{l}131.7119 \\
108.1515 \\
132.8575 \\
{[107.179]}\end{array}$ & $\begin{array}{l}1.02+9 \\
1.02+8 \\
6.26+8 \\
1.16+7 \\
\end{array}$ & $\begin{array}{l}1.859 \\
0.125 \\
1.159 \\
0.014 \\
\end{array}$ \\
\hline$(5 / 2,3 / 2) 4$ & $\begin{array}{l}(7 / 2,1 / 2) 3 \\
(5 / 2,1 / 2) 3 \\
(7 / 2,1 / 2) 4\end{array}$ & $\begin{array}{c}{[87.974]} \\
103.6662 \\
{[87.342]}\end{array}$ & $\begin{array}{l}7.38+6 \\
3.55+9 \\
3.07+6\end{array}$ & $\begin{array}{l}0.008 \\
5.142 \\
0.003\end{array}$ \\
\hline$(7 / 2,3 / 2) 4$ & $\begin{array}{l}(7 / 2,1 / 2) 3 \\
(5 / 2,1 / 2) 3 \\
(7 / 2,1 / 2) 4 \\
\end{array}$ & $\begin{array}{l}{[101.631]} \\
{[123.178]} \\
100.7742 \\
\end{array}$ & $\begin{array}{l}1.39+9 \\
3.34+6 \\
2.44+9 \\
\end{array}$ & $\begin{array}{l}1.935 \\
0.007 \\
3.339 \\
\end{array}$ \\
\hline$(7 / 2,1 / 2) 4$ & $\begin{array}{l}(7 / 2,1 / 2) 3 \\
(5 / 2,1 / 2) 3 \\
(7 / 2,1 / 2) 4 \\
\end{array}$ & $\begin{array}{l}132.2495 \\
{[171.202]} \\
130.8002\end{array}$ & $\begin{array}{l}1.08+9 \\
1.66+5 \\
6.53+8\end{array}$ & \begin{tabular}{|c}
2.546 \\
$7 \cdot 10^{-4}$ \\
1.507 \\
\end{tabular} \\
\hline$(7 / 2,3 / 2) 5$ & $(7 / 2,1 / 2) 4$ & 103.6219 & $3.55+9$ & 6.297 \\
\hline
\end{tabular}

Примечание. * Вычисленные значения длин волн $\lambda_{\text {calc }}$ заключены в квадратные скобки. ${ }^{* *} 3.39+9=3.3 \cdot 10^{9}$.

\section{Список литературы}

[1] Kramida A., Ralchenko Yu., Reader J., and NIST ASD Team. NIST Atomic Spectra Database (ver. 5.3). Электронный pecypc. Режим доступа: http://physics.nist.gov/asd

[2] Анисимова Г.П., Логинов А.В., Тучкин В.И. // Опт. и спектр. 2001. Т. 90. № 3.

[3] Логинов А.В., Тучкин В.И. // Опт. и спектр. 2001. Т. 90. № 5. C. 709.

[4] Sugar J., Kaufman V. // Phys. Rev. A. 1975. V. 12. N 3. P. 994.
Таблица 4. Длины волн $(\lambda, \mathrm{nm})$, вероятности $\left(A, \mathrm{~s}^{-1}\right)$ и силы осцилляторов $(g f)$ переходов $4 f^{13} 7 s \rightarrow 4 f^{13} 6 p$ в спектре W VII

\begin{tabular}{|c|c|c|c|c|}
\hline $4 f^{13} 7 s$ & $4 f^{13} 6 p$ & $\lambda_{\exp }[4], \lambda_{\text {calc }}^{*}, \mathrm{~nm}$ & $A, \mathrm{~s}^{-1}$ & $g f$ \\
\hline$(5 / 2,1 / 2) 2$ & \begin{tabular}{|l|}
$(5 / 2,3 / 2) 1$ \\
$(7 / 2,3 / 2) 2$ \\
$(5 / 2,1 / 2) 2$ \\
$(5 / 2,3 / 2) 2$ \\
$(7 / 2,3 / 2) 3$ \\
$(7 / 2,1 / 2) 3$ \\
$(5 / 2,3 / 2) 3$ \\
$(5 / 2,1 / 2) 3$ \\
\end{tabular} & $\begin{array}{l}69.7423 \\
{[62.969]} \\
{[61.783]} \\
71.1339 \\
{[63.120]} \\
{[55.453]} \\
71.6010 \\
61.4402 \\
\end{array}$ & $\begin{array}{c}1.48+9^{* *} \\
5.65+7 \\
5.30+8 \\
1.95+9 \\
1.09+8 \\
1.00+6 \\
1.25+9 \\
3.00+9 \\
\end{array}$ & $\begin{array}{c}0.539 \\
0.017 \\
0.152 \\
0.741 \\
0.033 \\
2 \cdot 10^{-4} \\
0.479 \\
0.849 \\
\end{array}$ \\
\hline$(7 / 2,1 / 2) 3$ & \begin{tabular}{|l|}
$(7 / 2,3 / 2) 2$ \\
$(5 / 2,1 / 2) 2$ \\
$(5 / 2,3 / 2) 2$ \\
$(7 / 2,3 / 2) 3$ \\
$(7 / 2,1 / 2) 3$ \\
$(5 / 2,3 / 2) 3$ \\
$(5 / 2,1 / 2) 3$ \\
$(5 / 2,3 / 2) 4$ \\
$(7 / 2,3 / 2) 4$ \\
$(7 / 2,1 / 2) 4$ \\
\end{tabular} & $\begin{array}{l}70.5562 \\
{[69.077]} \\
{[80.990]} \\
70.7607 \\
61.2456 \\
{[81.599]} \\
{[68.654]} \\
{[80.342]} \\
71.5617 \\
61.5320 \\
\end{array}$ & $\begin{array}{l}1.62+9 \\
6.21+7 \\
4.52+6 \\
1.69+9 \\
6.11+8 \\
1.00+5 \\
6.91+7 \\
1.13+5 \\
1.09+9 \\
2.94+9 \\
\end{array}$ & $\begin{array}{c}0.848 \\
0.031 \\
0.003 \\
0.890 \\
0.241 \\
7 \cdot 10^{-5} \\
0.034 \\
8 \cdot 10^{-5} \\
0.588 \\
1.167 \\
\end{array}$ \\
\hline$(5 / 2$, & \begin{tabular}{|l|}
$(7 / 2,3 / 2) 2$ \\
$(5 / 2,1 / 2) 2$ \\
$(5 / 2,3 / 2) 2$ \\
$(7 / 2,3 / 2) 3$ \\
$(7 / 2,1 / 2) 3$ \\
$(5 / 2,3 / 2) 3$ \\
$(5 / 2,1 / 2) 3$ \\
$(5 / 2,3 / 2) 4$ \\
$(7 / 2,3 / 2) 4$ \\
$(7 / 2,1 / 2) 4$ \\
\end{tabular} & $\begin{array}{l}{[62.880]} \\
61.7156 \\
71.0240 \\
{[63.031]} \\
{[55.384]} \\
71.4896 \\
61.3571 \\
70.5300 \\
{[63.671]} \\
{[55.608]} \\
\end{array}$ & $\begin{array}{l}5.59+7 \\
2.08+9 \\
2.71+8 \\
3.71+7 \\
3.17+6 \\
1.41+9 \\
1.38+9 \\
3.09+9 \\
2.13+6 \\
4.02+5 \\
\end{array}$ & \begin{tabular}{|c|}
0.023 \\
0.831 \\
0.143 \\
0.015 \\
0.001 \\
0.757 \\
0.544 \\
1.614 \\
$9 \cdot 10^{-4}$ \\
$1 \cdot 10^{-4}$ \\
\end{tabular} \\
\hline$(7$ & $\begin{array}{l}(7 / 2,3 / 2) 3 \\
(7 / 2,1 / 2) 3 \\
(5 / 2,3 / 2) 3 \\
(5 / 2,1 / 2) 3 \\
(5 / 2,3 / 2) 4 \\
(7 / 2,3 / 2) 4 \\
(7 / 2,1 / 2) 4 \\
(7 / 2,3 / 2) 5\end{array}$ & $\begin{array}{l}70.9085 \\
61.3571 \\
{[81.793]} \\
{[68.791]} \\
{[80.530]} \\
71.7131 \\
61.6436 \\
70.3377\end{array}$ & $\begin{array}{l}2.19+8 \\
2.34+9 \\
6.57+5 \\
7.30+6 \\
8.35+5 \\
1.44+9 \\
1.33+9 \\
2.97+9\end{array}$ & $\begin{array}{c}0.148 \\
1.191 \\
6.10^{-4} \\
0.005 \\
7.10^{-4} \\
1.001 \\
0.682 \\
1.979\end{array}$ \\
\hline
\end{tabular}

Примечание.* Вычисленные значения длин волн $\lambda_{\text {calc }}$ заключены в квадратные скобки. ${ }^{* *} 1.48+9=1.48 \cdot 10^{9}$.

[5] Wybourne B.G. Spectroscopic Properties of the Rare Earths. NY: Wiley, 1965.

[6] Cowan R.D. The Theory of Atomic Structure and Spectra. Berkeley: Univ. Calif. Press, 1981. 